\title{
Difference between Consensus Value of Participants' Results and Isotope-Dilution Mass Spectrometric Results in Proficiency Testing for Pesticide Residues in Husked Wheat
}

\author{
Takashi Yarita, ${ }^{\dagger}$ Takamitsu Otake, Yoshie Aoyagi, Masahiko Numata, and Akiko Takatsu \\ National Metrology Institute of Japan (NMIJ), National Institute of Advanced Industrial Science and \\ Technology (AIST), Umezono, Tsukuba 305-8563, Japan
}

\begin{abstract}
Proficiency testing was organized by the National Metrology Institute of Japan (NMIJ) as a measure of analytical competency in the quantification of pesticide residues in husked wheat powder. Seventy-one participants submitted analytical concentrations of the target pesticides (diazinon, fenitrothion, malathion, and etofenprox) along with details of the analytical method employed. Two types of assigned values were obtained for each target pesticide, i.e., the participants' analytical results and the results obtained by NMIJ based on isotope-dilution mass spectrometry (IDMS). The former values were lower than the latter due to the incomplete recovery yield of the target pesticides during the pretreatment process. The discrepancy between the two assigned values was particularly pronounced for malathion because of the longer duration of water-soaking used for the test samples. Two corresponding types of $z$-scores were then calculated to evaluate the analytical performance of the participants, where the $z_{1}$-score indicates the performance of a participant relative to all participants, and the $z_{2}$-score indicates the relative deviation of the analytical results of the participant from the IDMS value.
\end{abstract}

Keywords Proficiency testing, pesticide, assigned value, $z$-score, water-soaking, primary method of measurement

(Received December 7, 2015; Accepted January 12, 2016; Published May 10, 2016)

\section{Introduction}

Various pesticides are used to protect foods against pests and diseases. ${ }^{1}$ However, high levels of residual pesticides in food may result in adverse effects on human health. Therefore, the analysis of various residual pesticides in food is routinely performed worldwide, while ensuring that the reliability of these results is crucial for controlling the risk associated with pesticide residues.

Proficiency testing (PT) is an effective procedure in the implementation of an appropriate quality-assurance program and performance monitoring procedure for chemical analysis laboratories. ${ }^{2,3}$ For instance, the testing laboratories accredited by ISO/IEC 17025 are encouraged to participate in PT as a means of monitoring the validity of the test results from these laboratories. ${ }^{4}$ In addition, participating in PT assists participants to locate technical areas that require improvement. ${ }^{5,6}$ Consequently, PT has been routinely carried out by numerous providers in a wide variety of chemical quantitation fields, including the analysis of pesticide residues in food. ${ }^{7-9}$

In 2012, the National Metrology Institute of Japan (NMIJ) initiated a new PT program for the analysis of pesticide residues in food samples. ${ }^{10}$ The first round PT was carried out using the candidate soybean powder material, NMIJ CRM 7509-a, ${ }^{11}$ as the test sample. In the ensuing year, the second round PT was

† To whom correspondence should be addressed.

E-mail: t-yarita@aist.go.jp carried out using brown rice powder as the test sample. ${ }^{12}$ In this program, two types of $z$-scores are provided for each target pesticide. These scores are provided based on assigned values obtained from the participants' results, and results obtained by the NMIJ using isotope-dilution mass spectrometry (IDMS). ${ }^{13,14}$ Another feature of PT is that real crop samples contaminated with the target pesticides are used as the test samples to accurately evaluate the analytical process employed by the participants.

In 2014, the third round PT was carried out using husked wheat powder as the test sample. In this round, a significant deviation (about $30 \%$ on a relative basis) between the two assigned values was observed for malathion ( $S$-1,2bis(ethoxycarbonyl)ethyl $O, O$-dimethyl phosphorodithioate), which was one of the four target pesticides evaluated. Thus, additional experiments were performed to delineate the effect of the water-soaking conditions employed for pretreatment of the test samples on the observed concentration of malathion. This report summarizes the process and results of the 2014 PT. The origin of the difference between the assigned values for malathion is also discussed.

\section{Experimental}

Test samples

Diazinon $(O, O$-diethyl- $O$-2-isopropyl-6-methylpyrimidin-4-yl

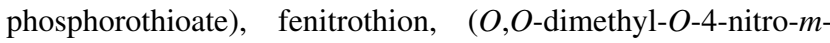
tolyl phosphorothioate), etofenprox (2-(4-ethoxyphenyl)-2- 
methylpropyl 3-phenoxybenzyl ether), and malathion were used as the target pesticides. Spraying liquid containing these pesticides at concentrations higher than the usual application concentrations was applied by a sprayer to wheat three times at 26,19 , and 7 days before harvest. The obtained husked wheat was air-dried and mixed with commercially available husked wheat (confirmed to have no detectable target pesticides) in the appropriate ratio to adjust the concentration of the pesticide contaminants. The mixture was then freeze pulverized, divided into 8 fractions, and further bottled into 240 amber glass bottles ( $20 \mathrm{~g}$ each, 30 bottles per fraction). The prepared samples were sterilized by $\gamma$-irradiation ( $15 \mathrm{kGy}$ ).

\section{Determination by the participants}

Seventy-two participants, most of which were Japanese food manufacturers, testing laboratories, or agricultural producers registered in this PT round. On May 7, 2014, the test samples ( 2 bottles) were sent to each participant by a delivery company using frozen transportation $\left(\leq-18^{\circ} \mathrm{C}\right)$. The participants were asked to store these samples at -30 to $-20^{\circ} \mathrm{C}$ in the dark, and then to perform duplicate analyses of the respective target pesticides using each of the two bottles. The participants were also asked to report the details of the analytical method they employed and the quantification conditions as well as the analytical results, and to submit the raw chromatograms using the prescribed sheet in Excel format. The deadline was set to July 26, 2014.

\section{IDMS determination at NMIJ}

Chemicals. High-purity standards of the target pesticides (Tracesure CRMs) were purchased from Wako Pure Chemical Industries (Osaka, Japan). The certified purity of these materials had been provided by NMIJ. ${ }^{15}$ High-purity standards of diazinon- $d_{10}$, fenitrothion- $d_{6}$, and etofenprox- $d_{5}$ were obtained from Hayashi Pure Chemical Industry (Osaka, Japan). A highpurity standard of malathion- $d_{6}$ was obtained from CDN Isotopes Inc. (Pointe-Claire, Canada). A high-purity standard of alachlor, which was used as a syringe spike, was obtained from GL Sciences (Tokyo, Japan). Acetonitrile, acetone, toluene, and anhydrous sodium sulfate, all of which were of Pesticide Residue and PCB Analysis grade, were obtained from Kanto Chemical (Tokyo, Japan). Reagent-grade sodium chloride, dipotassium hydrogen phosphate, and potassium dihydrogen phosphate were also obtained from Kanto Chemical. The water used for sample pretreatment was prepared with a Millipore (San Jose, CA) Milli-Q Gradient system at an output of 18.2 $\mathrm{M} \Omega \mathrm{cm}$.

Preparation of a surrogate solution, a syringe spike solution, and calibration solutions. The surrogate solution was gravimetrically prepared by dissolving the high-purity standards of diazinon- $d_{10}$, fenitrothion- $d_{6}$, malathion- $d_{6}$, and etofenprox- $d_{5}$ in acetone. The syringe spike solution was also gravimetrically prepared by dissolving a high-purity standard of alachlor in acetone. The pesticide solutions were prepared by mixing the individual high-purity standards of the target pesticides with acetone, followed by combining the solutions to prepare the pesticide-mixture solution. This solution was further mixed with the surrogate and syringe spike solutions to prepare the calibration solution. Three batches of the calibration solution were prepared by two experimenters, and the batches were cross-checked by GC-MS analysis under the conditions described in the subsequent subsection. Matrix-matched calibration solutions were then prepared by mixing the calibration solution with a cleaned-up extract of the blank wheat that was used for preparing the test samples. The final concentrations of the target and isotope-labeled pesticides in this solution were adjusted to be almost equal to those in the sample solutions that were prepared for GC-MS measurements. Analytical method. The pretreatment protocol prescribed in the Multiresidue Method for Agricultural Chemicals by GC/MS (Agricultural Products) ${ }^{16}$ (hereafter referred to as the "Multiresidue Method") was used after some modification. The test sample $(5 \mathrm{~g})$ was spiked with the surrogate solution $(0.5$ $\mathrm{mL})$. Water $(20 \mathrm{~mL})$ was added to the sample, which was then allowed to stand for $15 \mathrm{~min}$. The sample was homogenized with acetonitrile $(50 \mathrm{~mL})$ for $2 \mathrm{~min}$ and filtered with a cellulose filter. The residue on the filter was re-homogenized with acetonitrile $(20 \mathrm{~mL})$ for $2 \mathrm{~min}$ and the filtrates were combined. An approximately $40 \mathrm{~mL}$ aliquot of the crude extract was fractionated in a separatory funnel and shaken with sodium chloride (10 g) and $0.5 \mathrm{~mol} / \mathrm{L}$ phosphate buffer solution ( $\mathrm{pH} 7.0$, $20 \mathrm{~mL}$ ) for $10 \mathrm{~min}$. The obtained upper layer and acetonitrile $(2 \mathrm{~mL}$ ) were passed through an Agilent Technologies (Palo Alto, CA) Bond Elut C18 cartridge $(1 \mathrm{~g})$ that was conditioned in advance with acetonitrile $(10 \mathrm{~mL})$, and the entire volume of the eluate was collected. After dehydration with anhydrous sodium sulfate (approximately $10 \mathrm{~g}$ ), the extract was concentrated, dried, and subsequently dissolved in an acetonitrile/toluene mixture $(3: 1, \mathrm{v} / \mathrm{v} ; 2 \mathrm{~mL})$. This extract was further purified using a Supelco (Bellefonte, PA) ENVI-Carb/LC-NH2 cartridge $(500 \mathrm{mg} / 500 \mathrm{mg})$ that was conditioned in advance with an acetonitrile/toluene mixture $(3: 1, \mathrm{v} / \mathrm{v} ; 10 \mathrm{~mL})$. An acetonitrile/ toluene mixture $(3: 1, \mathrm{v} / \mathrm{v} ; 20 \mathrm{~mL})$ was then passed through the cartridge, and the eluate was subsequently concentrated and dried. The sample solution was prepared by dissolving the dried residue in the syringe spike solution $(1.0 \mathrm{~mL})$.

The target pesticides in the sample solution were quantified by GC-MS using the same apparatus and analytical conditions employed in the previous study, ${ }^{11}$ with the exception that the monitored ions $(\mathrm{m} / \mathrm{z})$ were set as follows: diazinon, 304; diazinon- $d_{10}, 314$; fenitrothion, 277 ; fenitrothion- $d_{6}, 283$; malathion, 158; malathion- $d_{6}, 164$; etofenprox, 163; etofenprox- $d_{5}, 168$; alachlor, 160.

\section{Establishment of assigned values}

Two assigned values were established for each target pesticide. Assigned value $1\left(X_{\mathrm{m}}\right)$ was obtained from the analytical results of the participants as follows: outliers were excluded using the Cochran test and Grubbs' test, and the median of the included results was then calculated as $X_{\mathrm{m}}$. The normalized interquartile range $(N I Q R)$ was simultaneously calculated by multiplying the interquartile range (the difference of quartile 3 and quartile 1 ) of the included analytical results by 0.743 . The assigned value 2 ( $X_{\text {IDMS }}$ ) was obtained from the analytical results based on IDMS. The predicted reproducibility standard deviation $\left(P S D_{\mathrm{R}}\right)$, which is the expected inter-laboratory precision for each target pesticide, was also calculated by using the Horwitz equation or the Thompson-modified Horwitz equation in accordance with the concentration range of the $X_{\mathrm{IDMS}}$ value. ${ }^{17}$

\section{Calculation of z-scores}

The performance of each participant was evaluated by applying the $z$-score approach in accordance with the ISO/IEC $17043,{ }^{18}$ ISO $13528,{ }^{19}$ and the International Harmonized Protocol for the Proficiency Testing of Analytical Chemistry Laboratories ${ }^{3}$ methods. Two kinds of $z$-scores $\left(z_{1}\right.$ and $\left.z_{2}\right)$ were calculated using the following equations:

$$
z_{1}=\frac{x_{\mathrm{i}}-X_{\mathrm{m}}}{N I Q R}
$$


Table 1 Summary of the analytical methods and quantification conditions used by the participants

\begin{tabular}{|c|c|c|c|c|c|c|c|c|c|c|c|c|}
\hline \multirow[b]{2}{*}{ Pesticide } & \multirow[b]{2}{*}{$\begin{array}{l}\text { Number of } \\
\text { participants }\end{array}$} & \multicolumn{5}{|c|}{ Extraction and clean-up method } & \multicolumn{6}{|c|}{ Measurement method } \\
\hline & & $\begin{array}{l}\text { Multiresidue } \\
\text { Method }^{\text {a }}\end{array}$ & QuEChERS & STQ & SFE & Others & GC-MS & $\begin{array}{l}\text { GC-MS/ } \\
\text { MS }\end{array}$ & $\begin{array}{r}\mathrm{GC} \\
\text { pho } \\
\mathrm{d}\end{array}$ & $\begin{array}{l}\text { th flame } \\
\text { metric } \\
\text { ector }\end{array}$ & LC-MS & $\begin{array}{c}\text { LC-MS/ } \\
\text { MS }\end{array}$ \\
\hline Diazinon & 70 & 38 & 4 & 19 & 6 & 3 & 34 & 30 & & 2 & 0 & 4 \\
\hline Fenitrothion & 68 & 38 & 3 & 18 & 6 & 3 & 34 & 30 & & 2 & 0 & 2 \\
\hline Malathion & 69 & 37 & 4 & 19 & 6 & 3 & 34 & 28 & & 2 & 0 & 5 \\
\hline \multirow[t]{3}{*}{ Etofenprox } & 64 & 33 & 4 & 19 & 6 & 2 & 32 & 30 & & 0 & 1 & 1 \\
\hline & \multicolumn{10}{|c|}{ Matrix-matching of calibration solution } & \multicolumn{2}{|c|}{$\begin{array}{l}\text { Collection with } \\
\text { recovery yield }\end{array}$} \\
\hline & \multicolumn{2}{|c|}{$\begin{array}{l}\text { With polyethylene } \\
\text { glycol (PEG) }\end{array}$} & \multicolumn{3}{|c|}{$\begin{array}{c}\text { With clean-up extract of } \\
\text { blank wheat }\end{array}$} & \multicolumn{2}{|c|}{$\begin{array}{l}\text { With PEG and clean-up } \\
\text { extract of blank wheat }\end{array}$} & \multicolumn{2}{|c|}{$\begin{array}{l}\text { With other } \\
\text { substance }\end{array}$} & $\begin{array}{l}\text { Not } \\
\text { done }\end{array}$ & Done & $\begin{array}{l}\text { Not } \\
\text { done }\end{array}$ \\
\hline (Diazinon) & & 27 & 16 & & & & 2 & 4 & & 21 & 4 & 66 \\
\hline (Fenitrothion) & & 27 & 15 & & & & 2 & 2 & & 20 & 4 & 64 \\
\hline (Malathion) & & 27 & 16 & & & & 2 & 2 & & 20 & 4 & 65 \\
\hline (Etofenprox) & & 27 & 14 & & & & 2 & 2 & 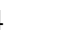 & 17 & 3 & 61 \\
\hline
\end{tabular}

a. Multiresidue Method for Agricultural Chemicals by GC/MS (Agricultural Products). ${ }^{16}$

$$
z_{2}=\frac{x_{\mathrm{i}}-X_{\mathrm{IDMS}}}{P S D_{\mathrm{R}}}
$$

where $x_{\mathrm{i}}$ represents the analytical result for the target pesticide of the individual participants.

\section{Results and Discussion}

Assessment of homogeneity and stability of the test samples

The homogeneity of the test samples was evaluated by quantifying the target pesticides in two sub-samples taken from ten bottles (bottles No. 1 and No. 240, and eight other bottles that were randomly selected from the 30 bottles prepared from the individual fractions). A one-way analysis of variance (ANOVA) test of the observed results (data not shown) was carried out according to the ISO Guide 35 method, ${ }^{20}$ where the inhomogeneities of diazinon, fenitrothion, and malathion were found to be statistically significant. However, the relative value of the standard deviations between bottles $\left(s_{\mathrm{bb}}\right)$ for diazinon, fenitrothion, malathion, and etofenprox were $0.557,1.17,3.66$, and $0.424 \%$, respectively; the $s_{\mathrm{bb}}$ values are significantly smaller than the expected $N I Q R$ and $P S D_{\mathrm{R}}$ values (the results are discussed below). The repeatability of the method $\left(u_{\mathrm{bb}}\right)$ was also evaluated according to the ISO Guide 35 method; the results were as follows: $0.224 \%$ for diazinon, $0.498 \%$ for fenitrothion, $1.25 \%$ for malathion, and $0.448 \%$ for etofenprox relative to the analytical values. Consequently, the uncertainty due to inhomogeneity was treated by using either $s_{\mathrm{bb}}$ or $u_{\mathrm{bb}}$, depending on which had the larger value.

The $s_{\text {bb }}$ value for malathion was relatively higher than that obtained for the other three pesticides; this was because the analytical results obtained for the contents of the two bottles that were individually selected from the 3rd fraction group and the 6th fraction group were comparatively lower than those of the other 8 bottles. To reduce the influence of any inhomogeneity of the test samples on the evaluation of the participants' performance, the test samples prepared from these two fraction groups were not used. Furthermore, the participants received two bottles collected from different fraction groups, and were asked to perform duplicate analyses of the respective target pesticides using the different bottles.

At NMIJ, the test samples were stored at -30 to $-20^{\circ} \mathrm{C}$ in the dark (according to instructions given to the participants) for the duration of the analytical period assigned to the participants; the changes in the concentration of the target pesticides were monitored during this period. A one-way ANOVA test of the observed results (data not shown) indicated that the difference in these data points was significant for diazinon and etofenprox. However, the calculated standard deviations between the data points $(1.35 \%$ for diazinon, $0.308 \%$ for fenitrothion, $2.42 \%$ for malathion, and $1.62 \%$ for etofenprox relative to the analytical values) appeared to be much lower than the expected NIQR and $P S D_{\mathrm{R}}$ values. Therefore, the pesticide concentrations of the test samples were expected to remain constant if the participants stored the samples under the instructed conditions. The observed standard deviations were used as the standard uncertainty due to instability.

\section{Analytical methods and overall results of the participants}

Analytical results were submitted by 71 participants. Answers to the questionnaire concerning the analytical method and quantification conditions are summarized in Table 1. More than half of the participants utilized the Multiresidue Method as the pretreatment method. Most of the other participants applied rapid pretreatment methods, such as the quick, easy, cheap, effective, rugged, and safe (QuEChERS) method, the solid phase extraction technique along with the QuEChERS (STQ) method, ${ }^{21}$ and supercritical fluid extraction (SFE). On the other hand, most participants did not correct their observed analytical concentrations with the recovery yields of the target pesticides during the pretreatment process, probably because the Multiresidue Method does not require this correction if the recovery yield of the target pesticide in a spike-and-recovery test meets the criterion $(70-120 \%)$ of the corresponding validation guideline. ${ }^{22}$

For diazinon, fenitrothion, and etofenprox, one participant reported analytical concentrations that were approximately three orders of magnitude lower than the median of the other participants' results; this discrepancy is thought to arise from an 
Table 2 Assigned value 1 and other fundamental statistics ${ }^{\mathrm{a}}$

\begin{tabular}{lcccc}
\hline \multicolumn{1}{c}{ Pesticide } & Quartile 1 & $\begin{array}{c}\text { Assigned } \\
\text { value 1 }\left(X_{\mathrm{m}}\right)\end{array}$ & Quartile 3 & NIQR \\
\hline Diazinon & 49.0 & 57.0 & 67.0 & 13.3 \\
Fenitrothion & 214 & 249 & 289 & 55.2 \\
Malathion & 32.9 & 39.4 & 49.2 & 12.1 \\
Etofenprox & 95.0 & 110 & 122 & 20.0 \\
\hline
\end{tabular}

a. Values are presented in units of $\mu \mathrm{g} / \mathrm{kg}$.

error in converting the units, and these results were rejected as outliers for further statistical treatment. The analytical results for diazinon and fenitrothion reported by another participant were rejected based on the results of the Cochran test. Relatively higher analytical results were reported by one participant for diazinon, three participants for fenitrothion, and four participants for malathion and etophenprox; these results were also rejected as outliers based on the Grubbs' test. The residual results showed unimodal distribution for each target pesticide. The participants' analytical results were also surveyed in order to evaluate the influence of the matrix-matching procedure used for the calibration solution and the influence of the extraction and clean-up methods utilized on the distribution of the results; no statistically significant differences were observed in either case (data not shown). The differences of these parameters in the analytical concentration is considered to be smaller than the variation of the values among laboratories.

\section{Assigned value 1}

Many Japanese PT providers use the median of the participants' results as the assigned values because this approach is simple and considered to be robust. ${ }^{23}$ Thus, in the current PT program, Assigned value 1 was obtained based on this approach. The fundamental statistics (including the $X_{\mathrm{m}}$ and NIQR values) of the four target pesticides were calculated after outlier tests, as shown in Table 2. The ratio of the $X_{\mathrm{m}}$ values to the corresponding Japanese MRLs ${ }^{24}$ were $57 \%$ for diazinon, $2.5 \%$ for fenitrothion, $0.5 \%$ for malathion, and $22 \%$ for etophenprox. The relative $N I Q R$ values to the corresponding $X_{\mathrm{m}}$ values for fenitrothion, diazinon, and etophenprox were 22.2, 23.3, and $18.2 \%$, respectively. It seemed that most participants could quantify these pesticides with good accuracy, even if the concentration of which in the test sample was much lower than the corresponding MRL value. The relative $N I Q R$ value to the corresponding $X_{\mathrm{m}}$ value for malathion $(30.7 \%)$ was considerably higher than those for the other three pesticides; the possible reason for this is described later.

\section{Assigned value 2}

The target pesticides were quantified by IDMS using matrixmatched calibration solutions. The reason that matrix-matching was performed is because the impact of the matrix effect on the target pesticides in the matrix-free calibration solution was different from that on the corresponding isotope-labeled internal standards. ${ }^{25}$ Because the linearity of the GC-MS response was good over the concentration range of the sample solutions, each analytical data point was obtained by the one-point calibration method using

$$
C=\frac{R_{\text {sample }}}{R_{\text {cal }}} \times \frac{F_{\text {ext }} \times F_{\text {cal }} \times M_{\text {cal }} \times C_{\text {cal }} \times P \times M_{\text {sur(sample) }}}{M_{\text {sample }} \times M_{\text {sur(cal })}},
$$

where $C$ is the analytical concentration of the target pesticide in
Table 3 Assigned value 2 with combined standard uncertainty and $P S D_{\mathrm{R}}{ }^{\mathrm{a}}$

\begin{tabular}{lccc}
\hline Pesticide & $\begin{array}{c}\text { Assigned } \\
\text { value } 2 \\
\left(X_{\text {IDMS }}\right)\end{array}$ & $\begin{array}{c}\text { Combined standard } \\
\text { uncertainty of } \\
\text { Assigned value 2 }\end{array}$ & $P S D_{\mathrm{R}}$ \\
\hline Diazinon & 66.0 & 1.5 & 14.5 \\
Fenitrothion & 284 & 10 & 54.9 \\
Malathion & 51.3 & 3.6 & 11.3 \\
Etofenprox & 123 & 4.7 & 26.9 \\
\hline
\end{tabular}

a. Values are presented in units of $\mu \mathrm{g} / \mathrm{kg}$.

Table 4 Uncertainty budget for quantification of etofenprox

\begin{tabular}{lclccc}
\hline $\begin{array}{c}\text { Source of } \\
\text { uncertainty }\end{array}$ & \multicolumn{1}{c}{$x_{\mathrm{i}}$} & \multicolumn{1}{c}{$u\left(x_{\mathrm{i}}\right)$} & Unit & $C_{\mathrm{i}}\left(=\frac{\partial f}{\partial x_{\mathrm{i}}}\right)$ & $\left|C_{\mathrm{i}}\right| u\left(x_{\mathrm{i}}\right)$ \\
\hline$F_{\text {ext }}$ & 1 & 0.026 & & 122.6 & 3.181 \\
$R_{\text {sample }} / R_{\text {cal }}$ & 1.0374 & 0.021 & & 118.2 & 2.478 \\
$F_{\text {cal }}$ & 1 & 0.0092 & & 122.6 & 1.133 \\
$M_{\text {cal }}$ & 0.79941 & 0.00014 & $\mathrm{~g}$ & 153.3 & 0.0217 \\
$C_{\text {cal }}$ & 3849.9 & 1.8 & $\mu \mathrm{g} / \mathrm{kg}$ & 0.03184 & 0.0572 \\
$P$ & 0.992 & 0.005 & $\mathrm{~kg} / \mathrm{kg}$ & 123.6 & 0.6178 \\
$M_{\text {spike(sample) }}$ & 0.3982 & 0.00029 & $\mathrm{~g}$ & 307.8 & 0.0896 \\
$M_{\text {sample }}$ & 5.0 & 0.00014 & $\mathrm{~g}$ & 24.52 & 0.0035 \\
$M_{\text {spike(cal) }}$ & 2.05774 & 0.00014 & $\mathrm{~g}$ & 59.57 & 0.0084 \\
$C$ and $u_{\mathrm{c}}(C)$ & 123 & 4.2 & $\mu \mathrm{g} / \mathrm{kg}$ & & \\
\hline
\end{tabular}

a. The explanation for each parameter is described in the text.

the test sample, $R_{\text {sample }}$ is the ratio of the peak area of the target pesticide/isotope-labeled pesticide observed for the sample solution, $R_{\text {cal }}$ is the ratio of the peak area of the target pesticide/ isotope-labeled pesticide observed for the matrix-matched calibration solution, $F_{\text {ext }}$ is the precision factor for the pretreatment process $(=1), F_{\text {cal }}$ is the precision factor for preparing the calibration solution (=1), $M_{\text {cal }}$ is the mass of the pesticide-mixture solution used for preparing the calibration solution, $C_{\text {cal }}$ is the concentration (dilution ratio) of the highpurity standard of the target pesticide in the pesticide-mixture solution, $P$ is the purity of the high-purity standard of the target pesticide, $M_{\text {sur(sample) }}$ is the mass of the surrogate solution added to the test sample, $M_{\text {sample }}$ is the mass of the test sample used for analysis, and $M_{\text {sur(cal) }}$ is the mass of the surrogate solution used for preparing the calibration solution. Here, the ratio of $R_{\text {sample }}$ to $R_{\text {cal }}\left(R_{\text {sample }} / R_{\text {cal }}\right)$ was evaluated as a single factor because during the GC-MS measurement, all of the sample solutions were analyzed at the same time using the matrix-matched calibration solution. Five independent measurements were carried out, and the mean of the observed concentrations was used as the $X_{\text {IDMs }}$ value, as shown in Table 3 .

The uncertainty corresponding to Assigned value 2 was also estimated. As the first step, the combined standard uncertainty of the mean of the analytical concentrations $\left[u_{\mathrm{c}}(C)\right]$ was obtained by estimating the standard uncertainty for the individual factors in Eq. (3), after which they were combined. The procedure for estimating the standard uncertainties of the individual factors was almost the same as that used during the production of our CRM. ${ }^{11}$ As an example of the results, the uncertainty budget for etofenprox is presented in Table 4 . The combined standard uncertainties of the analytical concentrations were $1.2 \mu \mathrm{g} / \mathrm{kg}$ for diazinon, $9.3 \mu \mathrm{g} / \mathrm{kg}$ for fenitrothion, and $2.8 \mu \mathrm{g} / \mathrm{kg}$ for malathion. The resulting uncertainty was further combined with 
the corresponding standard uncertainty due to an inhomogeneity and the standard uncertainty due to instability; the calculated value was used as the combined standard uncertainty associated with Assigned value 2. The results are summarized in Table 3. For all prepared samples (bottles) and during the test period assigned to the participants, Assigned value 2 was guaranteed to be within the concentration range obtained by multiplying this combined standard uncertainty by an appropriate coverage factor (such as $k=2$ ).

The $P S D_{\mathrm{R}}$ value was calculated for each target pesticide, as also shown in Table 3. The observed value is at least three times larger than the corresponding combined standard uncertainties for Assigned value 2.

\section{Comparison of the assigned values}

The $X_{\text {IDMs }}$ values for diazinon, fenitrothion, and etofenprox were, respectively, 16,14 , and $11 \%$ higher than the corresponding $X_{\mathrm{m}}$ values; similar tendencies were observed in our previous PT rounds, where the $X_{\text {IDMS }}$ values were 2.5 - $16 \%$ higher than the $X_{\mathrm{m}}$ values. ${ }^{10,12}$ As described above, most participants did not correct their observed analytical concentrations based on the recovery yields of the target pesticides obtained during the pretreatment process. At NMIJ, the recovery yields of isotopelabeled pesticides spiked onto the samples were monitored; the results (mean \pm S.D., $n=5)$ were as follows: $(83 \pm 4) \%$ for diazinon, $(83 \pm 6) \%$ for fenitrothion, $(74 \pm 7) \%$ for malathion, and $(90 \pm 10) \%$ for etofenprox. These values correspond to the recovery yield of the target pesticides obtained by the external calibration method in a conventional spike-and-recovery study. On the other hand, the recovery yield of the target pesticides does not affect the analytical concentration when the IDMS method is applied. The discrepancies between the two assigned values for the target pesticides are thought to arise from the difference in the impact of the recovery yield on the two analytical processes. For malathion, the $X_{\text {IDMS }}$ value was $30 \%$ higher than the $X_{\mathrm{m}}$ value; in this case, this significant difference may have another underlying cause, as discussed in the subsequent section.

\section{Effect of water-soaking on quantification of malathion}

Water-soaking is a treatment process in which the analytical sample is immersed in water prior to extraction. This process can enhance the efficiency of pesticide extraction from grain samples, especially for pesticides that have lower $\log P_{\text {ow }}$ values. ${ }^{26,27}$ On the other hand, some pesticides, including malathion, may undergo degradation during the water-soaking process. ${ }^{26}$ In the Multiresidue Method, grain samples are subjected to water-soaking for $15 \mathrm{~min}$. However, some participants changed the duration of this process. The watersoaking process was also utilized by most participants who utilized the STQ methods; however, the duration of the watersoaking process differed depending on the participants. The participants' analytical results derived from the use of both methods were surveyed to evaluate the influence of the duration of the water-soaking process; no statistically significant differences were observed in either case (data not shown). It seemed that the differences of the duration in the analytical concentration was smaller than the variation of the values among laboratories. Thus, NMIJ investigated the effect of the water-soaking conditions on the analytical concentration of the target pesticides in the test sample. Figure 1 shows the concentrations obtained with the variation of the water-soaking protocol using our IDMS method. In the experiments for obtaining Assigned value 2, the surrogate solution was added to the test sample before the water-soaking process; therefore, the
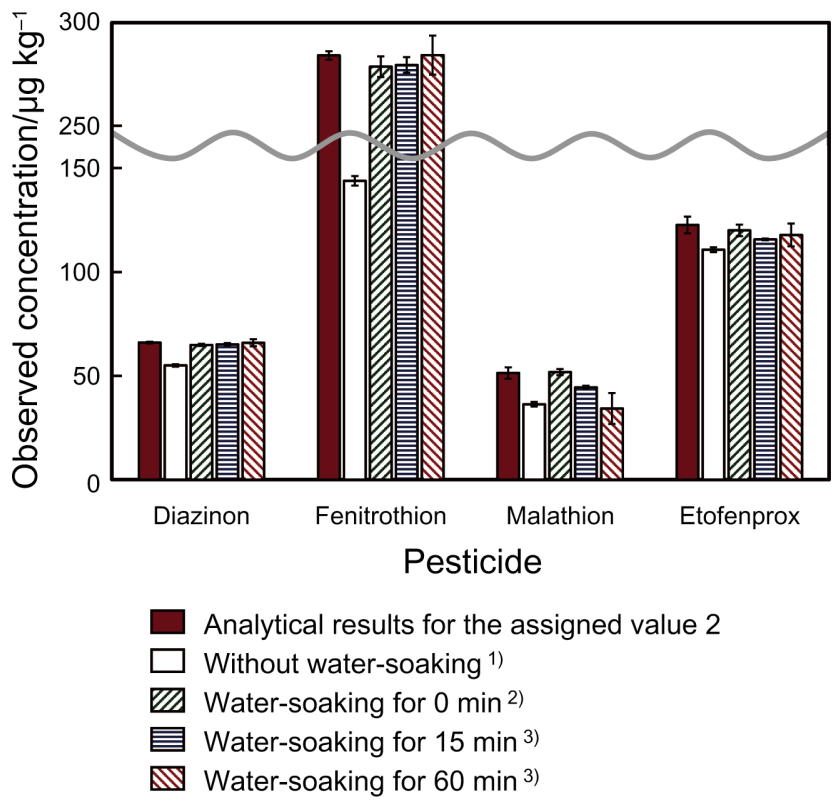

Fig. 1 Variation of the observed analytical concentration of the target pesticides based on the water-soaking conditions. Error bars show standard deviations ( $n=3$, except for the analysis for Assigned value 2 where $n=5$ ). 1) The surrogate solution was added to the test sample without water-soaking, after which homogenization with acetonitrile was continuously performed. 2) The surrogate solution and water were simultaneously added to the test sample, after which homogenization with acetonitrile was continuously performed. 3) After water-soaking for $15 \mathrm{~min}$ or $1 \mathrm{~h}$, the surrogate solution was added to the test sample and the sample was continuously homogenized with acetonitrile.

observed concentrations were considered not to have been influenced by the soaking. When extraction was performed without the water-soaking step, the observed concentrations of all target pesticides were $9.7-49 \%$ (in relative) lower than the corresponding concentrations for Assigned value 2. On the other hand, analytical results comparable to Assigned value 2 were obtained for all target pesticides when the sample was soaked just before the extraction. Moreover, the observed concentration of malathion decreased with an increase in the duration of soaking. These results suggest that when the Multiresidue Method or the STQ method was utilized for the analysis of the test samples, the water-soaking step was required to enhance the extraction efficiency for all target pesticides, but a longer duration of soaking resulted in lower concentrations of malathion. That is, it appears that this phenomenon contributes to the notably large differences between Assigned value 1 and Assigned value 2 for malathion. As described above, degradation during the water-soaking process for a sample might be a possible reason for the reduction in the concentration of malathion. However, the aqueous hydrolysis time required for $50 \%$ dissipation of the initial concentration (DT50) of malathion $\left(\mathrm{pH} 7,20^{\circ} \mathrm{C}\right)$ is reported to be 6.2 days ${ }^{28}$ which is much longer than the duration of the water-soaking process in the present study. Therefore, additional reasons may be necessary to explain this phenomenon.

\section{Assessment of the participants' performance}

For each participant, the $z_{1}$-scores and $z_{2}$-scores were calculated for the respective target pesticides. The $z_{1}$-score shows the analytical performance of each participant relative to 

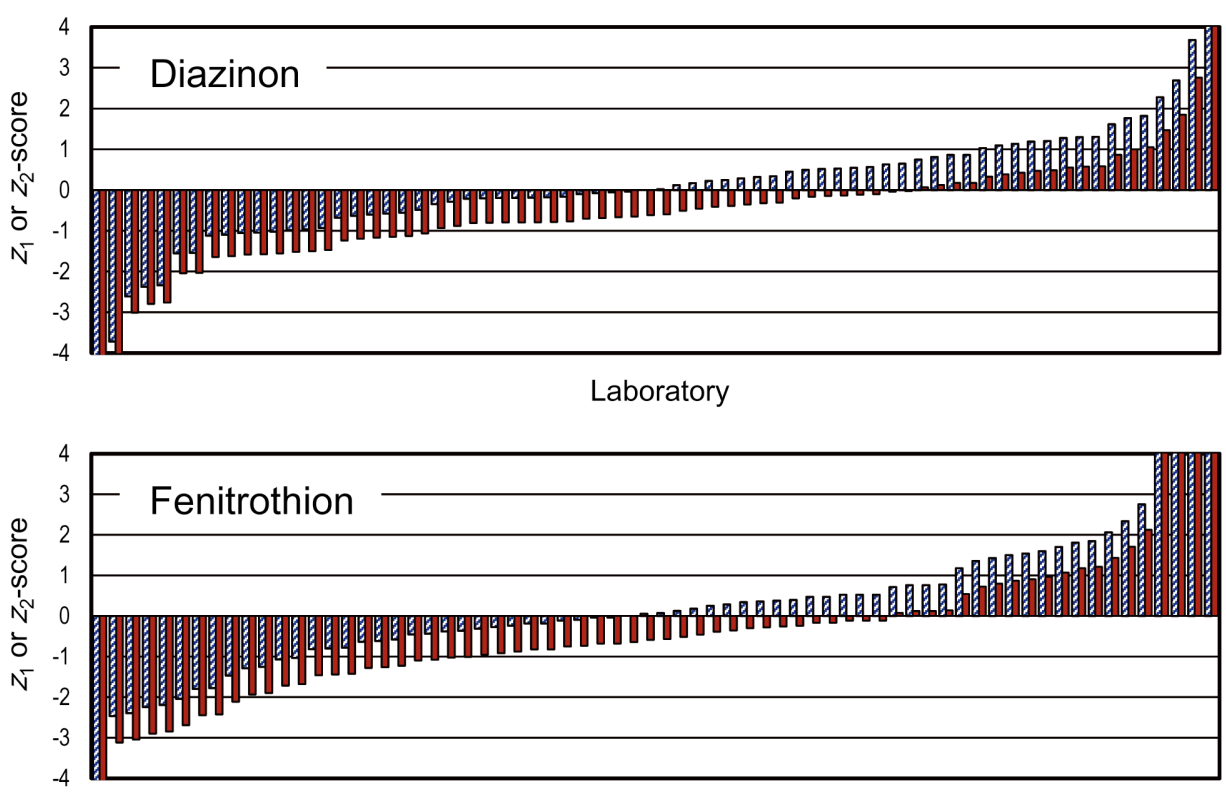

Laboratory

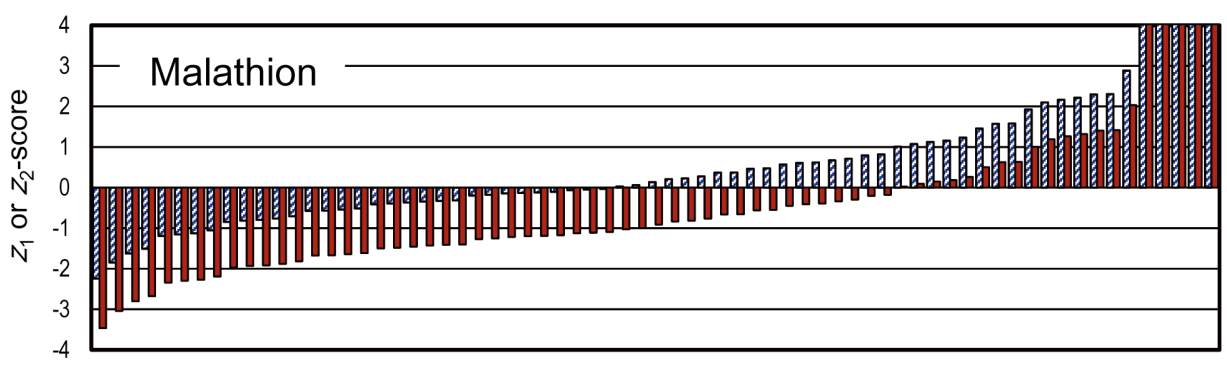

Laboratory

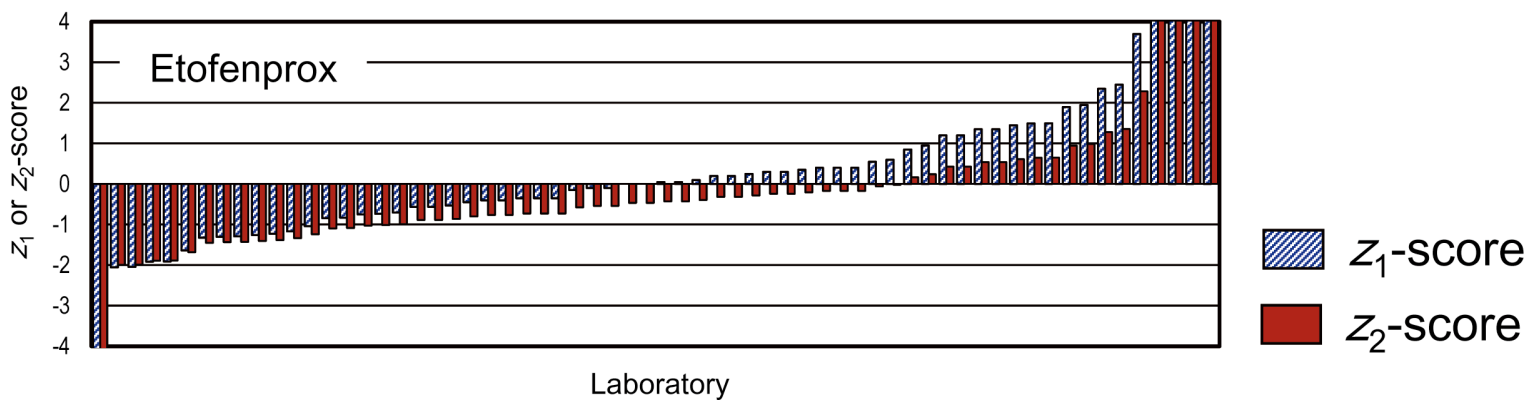

Fig. 2 Distribution of the participants' $z_{1}$ - and $z_{2}$-scores.

all participants in the present PT round. The $z_{2}$-score indicates the relative deviation of each participant's results from the IDMS value; this score is useful for evaluating the bias of the participants' analytical results. The distribution of these scores for all participants is shown in Fig. 2. Because Assigned value 2 was higher than the corresponding Assigned value 1, the $z_{2}$-score tended to be lower than the corresponding $z_{1}$-score. Based on the ISO/IEC 17043 protocol, ${ }^{18}$ the participants' performance was ranked into the following classes:

(1) $\left|z_{1}\right|,\left|z_{2}\right| \leq 2.0$, "satisfactory" performance,

(2) $2.0<\left|z_{1}\right|,\left|z_{2}\right|<3.0$, "questionable" performance, and

(3) $3.0 \leq\left|z_{1}\right|,\left|z_{2}\right|$, "unsatisfactory" performance.

The ratios of the participants having $\left|z_{1}\right|$ values ranking in the second or third classes were $13 \%$ for diazinon, $19 \%$ for fenitrothion, $17 \%$ for malathion, and $16 \%$ for etofenprox. The observed ratios were significantly higher than $5 \%$, which is the expected value when the participants' analytical results follow the normal distribution. The influence of the parameters shown in Table 1, such as the extraction and clean-up method, measurement method, matrix-matching of calibration solution, and collection with the recovery yield, on the participants' $z$-scores was evaluated; no significant differences in the scores were observed in any case. As already described, some of these parameters might cause differences in the analytical concentration. However, the variation of the participant's results that were obtained under the same analytical method and condition is considered to be larger than these differences. Thus, the quantification conditions and chromatograms of the participants who had poor $z$-score(s) were surveyed. As a result, a common technical problem among these participants could not be identified. However, one problem was confirmed, whereby a few participants quantified the target pesticide by 
chromatographic measurements outside of the dynamic range of the calibration curve; this could have caused the difference between their analytical concentration and the corresponding assigned values.

\section{Conclusions}

PT was carried out for the determination of four pesticide residues in husked wheat powder. The performance of seventyone participants was evaluated based on two types of assigned values. One of the notable features of the present round was that the Assigned value 1 for malathion was influenced by the water-soaking treatment process for the samples. Assigned value 2 , which was obtained from IDMS analysis, was not influenced by the analytical method and quantification conditions employed by the participants, including the conditions used for water-soaking of the test samples. The participants were instructed how to distinguish these assigned values in order to select the appropriate $z$-score based on their purpose for participating in the assessment.

Certain technical problems that may be sources of error influencing the analytical results were detected during assessment of the participants' results. These problems were discussed with the participants at the lecture held at the end of the present PT round, and hopefully measures will be implemented to improve their analytical methods or quality management protocol.

\section{Acknowledgements}

The authors thank KANSO Technos for assistance with preparation of the test samples. This work was partly supported by a Health and Labour Science Research Grant from the Ministry of Health, Labour, and Welfare.

\section{References}

1. F. P. Carvalho, Environ. Sci. Policy, 2006, 9, 685.

2. I. Taverniers, M. De Loose, and E. Van Bockstaele, Trends Anal. Chem., 2004, 23, 535.

3. M. Thompson, S. R. Ellison, and R. Wood, Pure Appl. Chem., 2006, 78, 145.

4. ISO/IEC 17025, "General Requirements for the Competence of Testing and Calibration Laboratories", 2005, International Organization for Standardization, Geneva.

5. P. Araujo and L. Frøyland, Anal. Chim. Acta, 2006, 555, 348.

6. S. Miyashita, K. Inagaki, S. Naito, K. Shindoh, T. Narukawa, Y. Zhu, T. Kuroiwa, A. Hioki, T. Fujimoto, and K. Chiba, Anal. Bioanal. Chem., 2013, 405, 8347.

7. P. Medina-Pastor, C. Rodríguez-Torreblanca, A. Andersson, and A. R. Fernández-Alba, Trends Anal. Chem., 2010, 29, 70

8. T. Watanabe, J. Food Hyg. Soc. Jpn., 2011, 52, J315.

9. M. Sykes, M. Thompson, and S. Reynolds, J. Agric. Food Chem., 2013, 61, 4205.

10. T. Yarita, T. Otake, Y. Aoyagi, T. Kuroiwa, M. Numata, and A. Takatsu, Talanta, 2015, 132, 269.

11. T. Yarita, T. Otake, Y. Aoyagi, Y. Kuroda, M. Numata, H. Iwata, M. Watai, H. Mitsuda, T. Fujikawa, and H. Ota, Talanta, 2014, 119, 255.

12. T. Otake, T. Yarita, Y. Aoyagi, M. Numata, and A. Takatsu, Anal. Bioanal. Chem., 2014, 406, 7337.

13. W. Richter, Accred. Qual. Assur., 1997, 2, 354.

14. M. J. T. Milton and T. J. Quinn, Metrologia, 2001, 38, 289.

15. T. Saito, T. Ihara, T. Miura, Y. Yamada, and K. Chiba, Accred. Qual. Assur., 2011, 16, 421.

16. Annex of the Director Notice (Syoku-an No 124001), "Analytical Methods for Residual Compositional Substances of Agricultural Chemicals, Feed Additives, and Veterinary Drugs in Food", 2005 (Amendment in 2006), Ministry of Health, Labour and Welfare, Tokyo.

17. M. Thompson, Analyst, 2000, 125, 385.

18. ISO/IEC 17043, "Conformity Assessment-General Requirements for Proficiency Testing", 2010, International Organization for Standardization, Geneva.

19. ISO 13528, "Statistical Methods for Use in Proficiency Testing by Interlaboratory Comparison”, 2005, International Organization for Standardization, Geneva.

20. ISO Guide 35, "Reference Materials-General and Statistical Principles for Certification", 2006, International Organization for Standardization, Geneva.

21. S. Hiramatsu, K. Nishiyama, S. Tokuhashi, T. Ashida, A. Kageyama, M. Takamiya, A. Nakamura, N. Takuma, and K. Nishimori, Rep. Pub. Hlth. Kochi, 2013, 59, 47.

22. Annex of the Director Notice (Syoku-An No 1224-1), "Guidelines for the Validation of Analytical Methods for Agricultural Chemical Residues in Food", 2010, Ministry of Health, Labour and Welfare, Tokyo.

23. M. Kubota, Y. Takata, K. Koizumi, Y. Ishibashi, R. Matsuda, Y. Matsumoto, K. Shikakume, A. Ono, M. Sakata, and K. Kakita, Bunseki Kagaku, 2008, 57, 393.

24. "Maximum Residue Limits (MRLs) List of Agricultural Chemicals in Foods", The Japan Food Chemical Research Foundation, http://www.m5.ws001.squarestart.ne.jp/ foundation/search.html.

25. T. Yarita, Y. Aoyagi, and T. Otake, J. Chromatogr. A, 2015, 1396, 109.

26. M. Saka, K. Iijima, Y. Koma, M. Fujita, Y. Odanaka, and Y. Kato, J. Pestic. Sci., 2006, 31, 431.

27. T. Otake, Y. Aoyagi, M. Matsuo, N. Itoh, and T. Yarita, $J$. Environ. Sci. Health B, 2008, 43, 390.

28. Pesticide Properties Database, "Malathion", University of Hertfordshire, http://sitem.herts.ac.uk/aeru/ppdb/en/Reports/ 421.htm. 